Article

\title{
High-Throughput Screening for Inhibitors of the SARS-CoV-2 Protease Using a FRET-Biosensor
}

\author{
Alistair S. Brown $₫$, David F. Ackerley $₫$ and Mark J. Calcott * \\ School of Biological Sciences, Victoria University of Wellington, Wellington 6012, New Zealand; \\ alistair.brown@vuw.ac.nz (A.S.B.); david.ackerley@vuw.ac.nz (D.F.A.) \\ * Correspondence: mark.calcott@vuw.ac.nz; Tel.: +64-27-866-3301
}

Received: 29 September 2020; Accepted: 12 October 2020; Published: 13 October 2020

check for updates

\begin{abstract}
The global SARS-CoV-2 pandemic started late 2019 and currently continues unabated. The lag-time for developing vaccines means it is of paramount importance to be able to quickly develop and repurpose therapeutic drugs. Protein-based biosensors allow screening to be performed using routine molecular laboratory equipment without a need for expensive chemical reagents. Here we present a biosensor for the 3-chymotrypsin-like cysteine protease from SARS-CoV-2, comprising a FRET-capable pair of fluorescent proteins held in proximity by a protease cleavable linker. We demonstrate the utility of this biosensor for inhibitor discovery by screening 1280 compounds from the Library of Pharmaceutically Active Compounds collection. The screening identified 65 inhibitors, with the 20 most active exhibiting sub-micromolar inhibition of $3 \mathrm{CL}^{\text {pro }}$ in follow-up $\mathrm{EC}_{50}$ assays. The top hits included several compounds not previously identified as $3 \mathrm{CL}^{\text {pro }}$ inhibitors, in particular five members of a family of aporphine alkaloids that offer promise as new antiviral drug leads.
\end{abstract}

Keywords: SARS-CoV-2; COVID-19; 3CLPro; cysteine protease; ebselen; apomorphine; aporphine

\section{Introduction}

Severe acute respiratory syndrome coronavirus 2 (SARS-CoV-2), the causative agent of the current coronavirus disease 2019 (COVID-19) outbreak, was first identified in late 2019. SARS-CoV-2 infections result in a range of symptoms including: loss of smell and taste, persistent cough and chest pain [1]. As of September 2020, there have been over 31 million confirmed cases of COVID-19 and approximately 970,000 deaths [2]. The spread of COVID-19 currently shows no signs of slowing and while several promising vaccines are in clinical development [3], all are yet to complete clinical trials and begin distribution to the general population. It is therefore of paramount importance to rapidly develop and/or repurpose safe and effective drugs to treat SARS-CoV-2 and reduce the global burden of this pandemic.

SARS-CoV-2 is a member of the Coronavirus family and belongs to the Betacoronavirus genus, the same genus as other notable human pathogens including severe acute respiratory syndrome coronavirus (SARS-CoV, with which it shares approximately $80 \%$ sequence identity [4]) and Middle East respiratory syndrome coronavirus. SARS-CoV-2 is a positive-sense, single stranded RNA virus with a genome consisting of approximately 30,000 nucleotides [5]. Two thirds of the genome consists of two open reading frames called ORF1a and ORF1b, which are translated using a programmed ribosomal frameshift into two polyproteins-pp1a and a C-terminus extended form pp1ab [6]. The two polyproteins contain 16 non-structural proteins, which have critical roles in viral replication. The two proteases, 3-chymotrypsin-like cysteine protease $\left(3 \mathrm{CL}^{\mathrm{pro}}\right)$, also known as the main protease, and papain-like protease are released auto-catalytically and cleave pp1a and pp1ab into the functional proteins [7] (Figure 1). $3 \mathrm{CL}^{\text {pro }}$ is a homodimer and is structurally highly similar to $3 \mathrm{CL}^{\text {pro }}$ from 
SARS-CoV [4,8] (Figure 1). 3CL pro recognises a cleavage site of X-(L/F/M)-Q $\downarrow$ (G/A/S)-X, wherein X represents any amino acid and $\downarrow$ represents the cleavage site [9]. No known human proteases recognise the same cleavage site, offering prospects for the identification of inhibitors with low toxicity profiles [9].

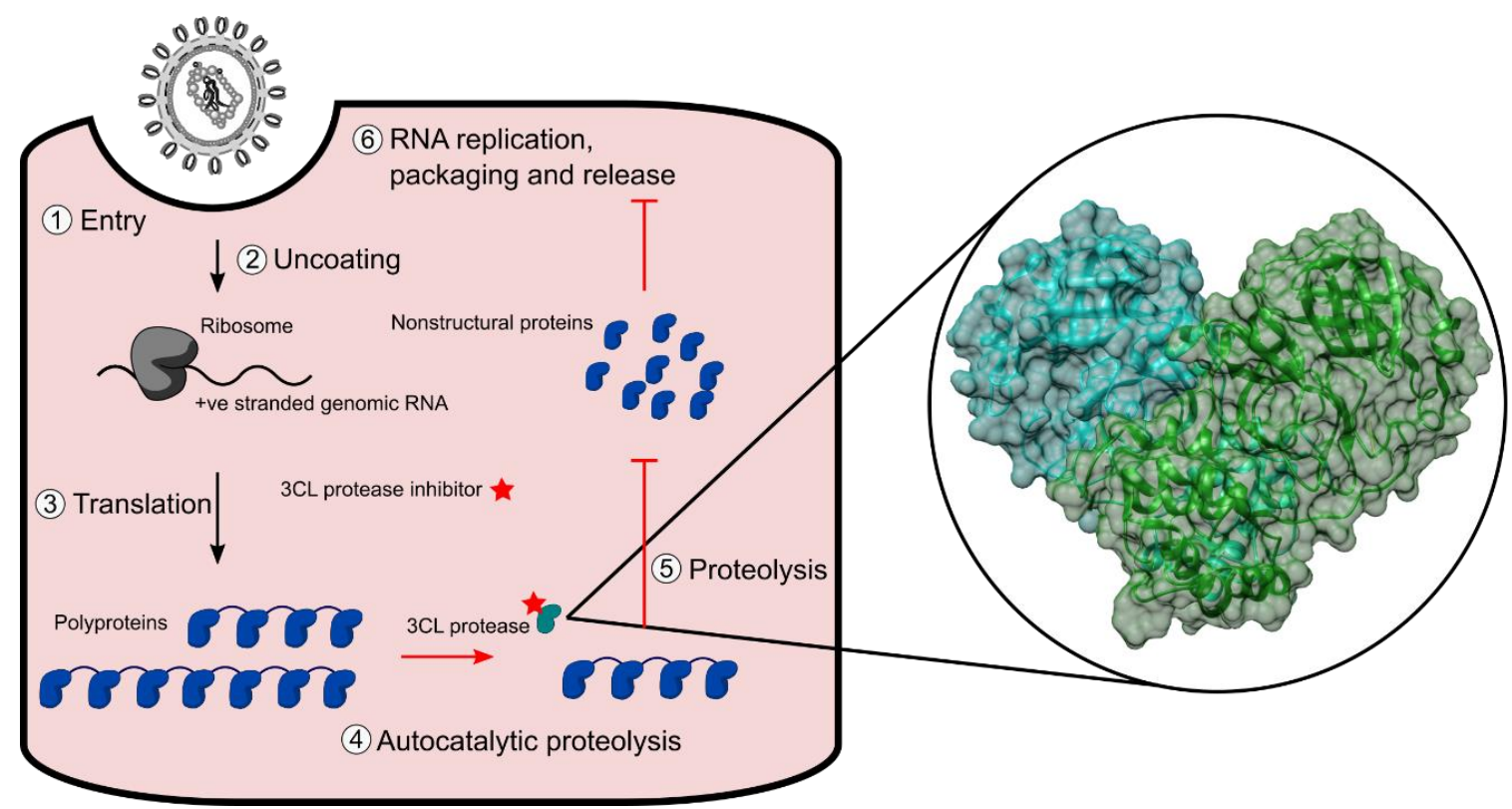

Figure 1. Schematic representation of the SARS-CoV-2 lifecycle in a host cell and the interruption by a $3 \mathrm{CL}^{\text {pro }}$ inhibitor. The insert is the crystal structure of $3 \mathrm{CL}^{\text {pro }}$ derived from PDB: 6M2Q.

Screening for inhibitors of SARS-CoV-2 3CL ${ }^{\text {pro }}$ has previously been conducted in high-throughput using a chemically-synthesised fluorophore and quencher separated by a cleavable peptide sequence [8]. Testing of 10,000 compounds with this probe identified seven hits, with ebselen being the strongest inhibitor. However, the specialised nature and cost of chemically synthesised probes makes them inaccessible to use in high-throughput screens for many facilities [10]. In contrast, protein-based biosensors can readily be prepared using equipment available in most molecular biology labs. A fluorescence resonance energy transfer (FRET)-based biosensor containing cyan-fluorescent protein (CFP) and yellow-fluorescent protein (YFP) as well as a luciferase-based biosensor have previously been used to determine the substrate specificity of 3CL pro from SARS-CoV [11] and MERS-CoV [12], respectively. We reasoned that it should be possible to use a similar design to construct a biosensor capable of accurately and sensitively reporting on drug-mediated inhibition of SARS-CoV-2 3CL ${ }^{\text {pro }}$.

Here we report our development, optimisation and application of a high-throughput screen and $\mathrm{EC}_{50}$ assay using a protein-based FRET-biosensor to identify inhibitors of 3CL ${ }^{\text {ro }}$ from SARS-CoV-2. The biosensor is easy to express and purify from Escherichia coli, making it cheap and accessible for molecular biology laboratories, and the screen is robust, with a high level of correlation between replicates.

\section{Results}

\subsection{Proteolysis of an eCFP-Venus Biosensor by SARS-CoV-2 3CL pro}

To create a FRET-based biosensor, the fluorescent proteins eCFP and Venus were selected because they generate a FRET signal with a large dynamic range [13]. A T7 promoter driven, N-terminus His 6 construct was made to express eCFP and Venus linked by the peptide sequence TSAVLQ $\downarrow S G F R K$. This peptide linker contains the cleavage site found immediately upstream to 3CL ${ }^{\text {pro }}$ in its native polyprotein [8] (Supplementary Figure S1). The purpose of the linker is to act as an on/off switch, holding the two fluorescent proteins in sufficiently close proximity for efficient FRET, with cleavage by $3 C^{\text {pro }}$ 
separating the proteins and thereby eliminating the signal at $528 \mathrm{~nm}$ (Figure 2A). The recombinant eCFP-Venus biosensor expressed well with no solubility or toxicity issues, and was purified using Ni-NTA chromatography from triplicate $400 \mathrm{~mL}$ E. coli BL21(DE3) cultures with a mean yield of $38.1 \pm 1.4 \mathrm{mg}$ of protein.

A $434 \mathrm{~nm}$

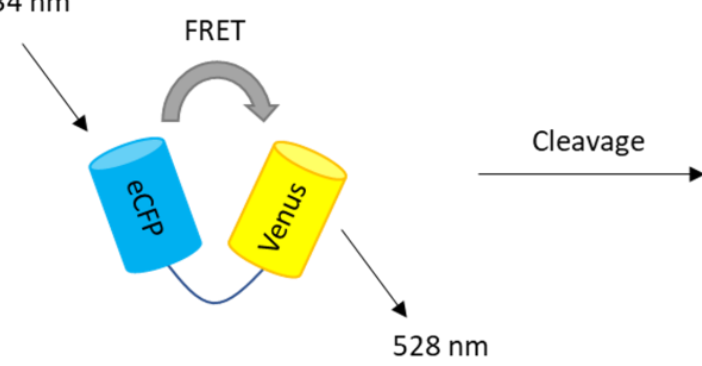

B

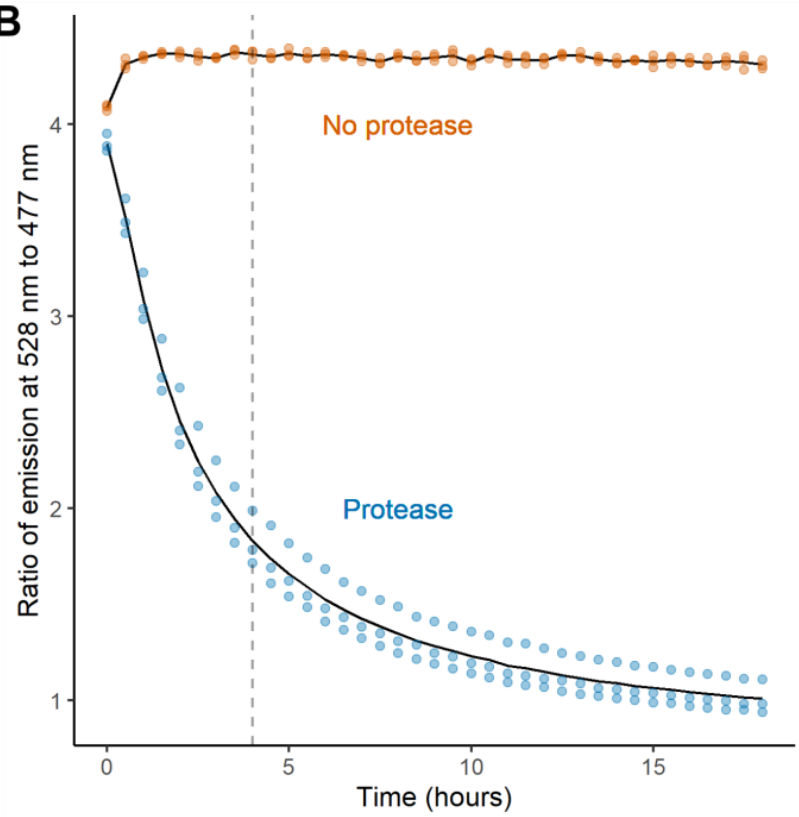

$434 \mathrm{~nm}$
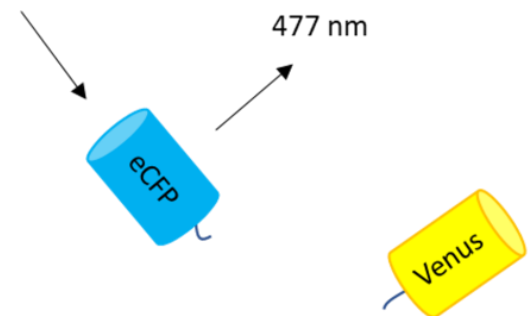

C

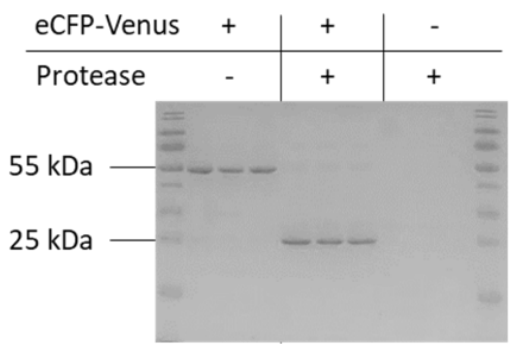

D

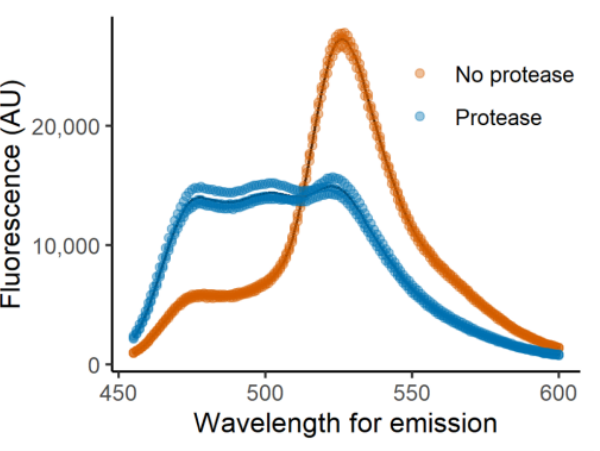

Figure 2. (A) When eCFP is excited at $434 \mathrm{~nm}$ in the intact biosensor, the close proximity of eCFP and Venus results in FRET emission from Venus at $528 \mathrm{~nm}$. Cleavage of the linker by SARS-CoV-2 3CL pro stops FRET occurring and emission now comes directly from eCFP at $477 \mathrm{~nm}$. (B) The dynamic ratio of emission from the FRET acceptor (Venus) and donor (eCFP)( $\left.\mathrm{R}_{528 / 477}\right)$ during treatment with (blue) or without (orange) SARS-CoV-2 3CL ${ }^{\text {pro }}$. The emission of the FRET donor $\left(\lambda_{\text {ex eCFP }} 477 \mathrm{~nm}\right.$ ) and acceptor $\left(\lambda_{\text {ex Venus }} 528 \mathrm{~nm}\right.$ ) after excitation at $434 \mathrm{~nm}$ were measured at $30 \mathrm{~min}$ intervals and used to calculate the $\mathrm{R}_{528 / 477}$. A dashed line at $4 \mathrm{~h}$ indicates the time used in endpoint assays for subsequent high-throughput screening. (C) A 12\% SDS-PAGE gel of samples taken from the reactions measured in Panel B at $18 \mathrm{~h}$. Each well in the SDS-PAGE gel contained a $12 \mu \mathrm{L}$ sample taken from a $200 \mu \mathrm{L}$ reaction. The presence of the eCFP-Venus biosensor and protease is indicated above the gel. Bands present at $55 \mathrm{kDa}$ are consistent with the eCFP-Venus biosensor, including intact linker and $\mathrm{His}_{6}$-tag (predicted mass of $56 \mathrm{kDa}$ ), and bands just above $25 \mathrm{kDa}$ are consistent with monomers of similarly sized eCFP (predicted mass of $27.5 \mathrm{kDa}$ ) and Venus (28.5 kDa). (D) Emission spectra of the eCFP-Venus biosensor after $18 \mathrm{~h}$ of treatment with (blue) and without (orange) protease. Experiments were performed in triplicate using separately purified eCFP-Venus biosensor and SARS-CoV-2 3CL ${ }^{\text {pro }}$. The average between replicates is represented by a line and all datapoints are shown as dots. 
To assess whether treatment with protease reduced FRET in a quantifiable fashion, reactions containing $500 \mathrm{nM}$ of the eCFP-Venus biosensor and $25 \mathrm{nM}$ of SARS-CoV-2 3CL ${ }^{\text {pro }}$ were incubated at $30^{\circ} \mathrm{C}$. The ratio of the emission maxima of the acceptor (Venus) to donor (eCFP) (R $\left.{ }_{528 / 477}\right)$ was calculated from measurements taken at 30-min intervals (Figure 2B). This showed a high signal stability in the absence of protease and a time-dependent decrease following protease treatment. After $18 \mathrm{~h}$ incubation, samples were analysed using SDS-PAGE (Figure 2C). The replicates without protease produced a band corresponding to intact eCFP-Venus biosensor. Treatment with protease caused this band to almost completely disappear and a band corresponding to the similarly sized monomers of eCFP and Venus to appear. Consistent with emission from Venus diminishing as the linker between eCFP and Venus was cleaved, complete spectra recorded at $18 \mathrm{~h}$ showed that protease treatment had caused a large decrease at $528 \mathrm{~nm}$, i.e., the emission maximum of Venus (Figure 2D). Collectively, these data indicated that the concentration of the eCFP-Venus biosensor used in these assays allowed robust measurement of protease activity and confirmed its suitability for high-throughput screening. Given our average yield of $38 \mathrm{mg}$ eCFP-Venus biosensor per $400 \mathrm{~mL}$ culture, this concentration allows for approximately 6800 reactions to be performed from a single culture.

\subsection{Characterisation and Application of the eCFP-Venus Biosensor for High Throughput Screening}

As a proof of concept, the eCFP-Venus FRET-biosensor was used to screen the library of pharmacologically active compounds (LOPAC ${ }^{\circledR 1280}$, Sigma-Aldrich, St. Louis, MO, USA) for 3CL pro inhibitors, on the presumption that an inhibitor would block cleavage and result in high $\mathrm{R}_{528 / 477}$ values, similar to the no protease controls from Figure 2B-D. An endpoint of $4 \mathrm{~h}$ was selected for measuring inhibition (Figure 2B), seeking to maximise the difference between digested and undigested eCFP-Venus biosensor while allowing the detection of weaker inhibitors by not letting the reaction run to completion. The LOPAC ${ }^{\circledR 1280}$ compounds were screened in duplicate at $5 \mu \mathrm{M}$ in 96 -well plates. Each plate also contained eight no-protease controls (representing 100\% protease inhibition) and eight no-inhibitor controls representing 0\% inhibition (Supplementary Figure S2).

We first assessed the quality of the data using the metric $Z^{\prime}$, which is based on the separation of positive and negative controls and is a key measure of quality for high-throughput screens [14]. A Z' value above 0.5 is considered an excellent result and represents 12 standard deviations of separation between the controls. Here, the relative percentage inhibition for the no protease $(M=100.00$, $\mathrm{SD}=2.07)$ and protease controls $(\mathrm{M}=0.00, \mathrm{SD}=1.14)$ from each plate revealed a clear separation band with a $Z^{\prime}$ of 0.9 (Figure 3). This confirmed the signal distinction was more than sufficient for high-throughput screening.

We next examined the consistency between duplicate wells for each of the LOPAC ${ }^{\circledR 1280}$ compounds, observing a high level of reproducibility with an $\mathrm{R}^{2}$ value of 0.97 (Figure 4A; Supplementary Data S1). The mean level of inhibition was close to $0 \%$ for most compounds. Setting a threshold of $19 \%$ inhibition, equivalent to a $Z^{\prime}$ score of 0.5 , identified 65 compounds that were inhibiting 3CLpro (Figure 4B). The library was then counter-screened without protease to assess the frequency at which compounds might interfere with FRET (Figure 4C). None of the 65 hits were identified as inhibiting FRET and, of the 1,280 compounds screened, only three (NF 023, anthrapyrazolone and dipyridamole) caused a large reduction in fluorescence. The high $\mathrm{R}^{2}$ value between replicates shows the assay to be robust at identifying hits, and screening in the absence of protease confirms that the false positive rate for compounds interfering with FRET is low and can be rapidly eliminated by counter screening in the absence of protease. 


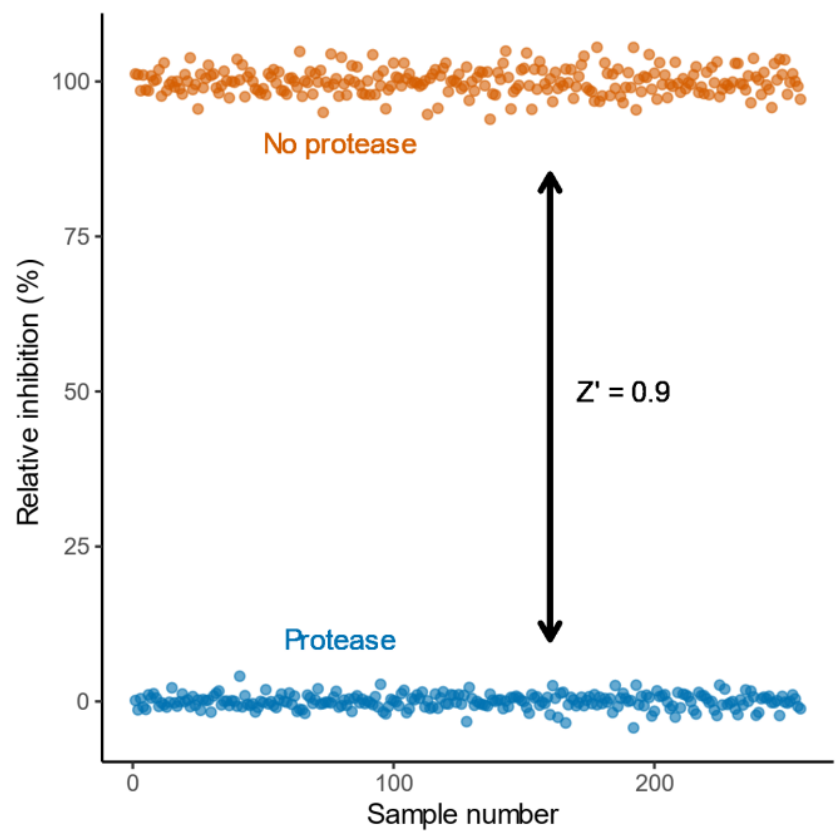

Figure 3. Separation of the positive and negative controls used to calculate relative inhibition of protease. Relative inhibition was calculated using the average $\mathrm{R}_{528 / 477}$ for the protease treated controls as $0 \%$ inhibition and the average $R_{528 / 477}$ for untreated controls as $100 \%$ inhibition. The data are the 256 no protease controls and 256 protease controls collected from all plates when screening the LOPAC ${ }^{\circledR 1280}$ in duplicate. All datapoints are shown.
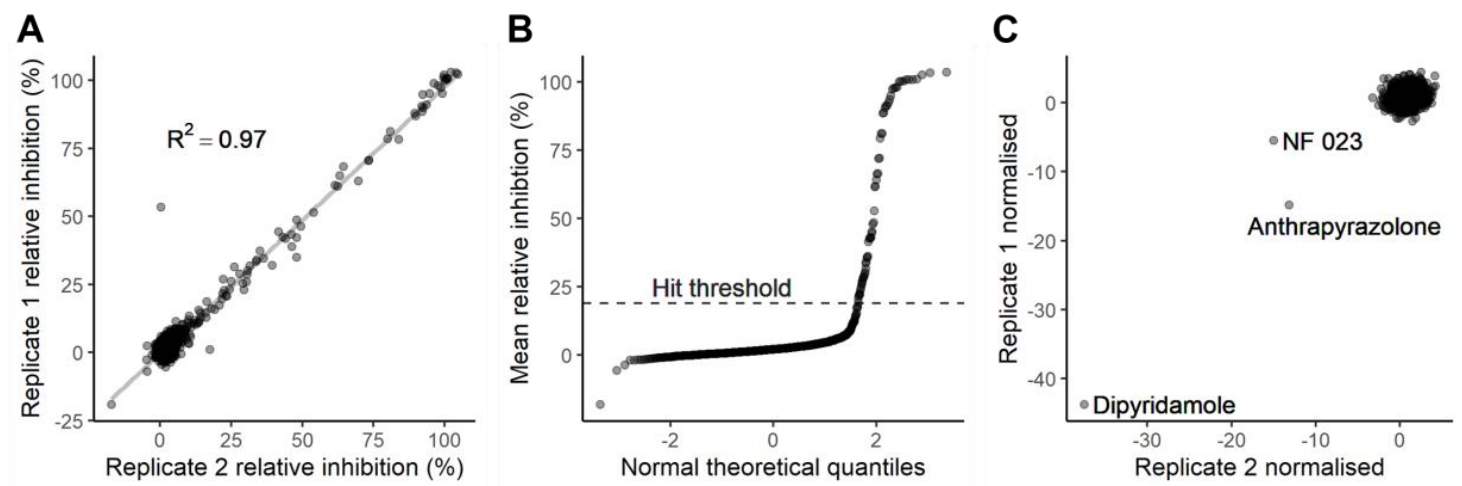

Figure 4. Screening the LOPAC ${ }^{\circledR 1280}$ in duplicate. (A) Consistency between duplicates during screening. Each axis represents one replicate. The relative inhibition of protease activity for compounds was calculated as per Figure 3. (B) A quantile-quantile plot showing mean relative inhibition versus normal theoretical quantiles. The $19 \%$ relative inhibition used as a threshold for hit selection is indicated with a dashed line. (C) The effect of compounds on the $R_{528 / 477}$ in the absence of protease. Each axis represents one replicate and $\mathrm{R}_{528 / 477}$ was normalised for each plate using sixteen eCFP-Venus biosensor (no protease) controls. Duplicate experiments were performed using separately purified eCFP-Venus biosensor and SARS-CoV-2 3CL pro, and all datapoints are shown.

The 65 compounds above the 19\% inhibition threshold were tested further in dose-response assays. All compounds exhibited a dose-response and the top 22 were found to have an $\mathrm{EC}_{50}$ of below $1 \mu \mathrm{M}$ (Supplementary Figure S3; Supplementary Table S1). Whereas the primary screen contained Triton X-100 at $0.01 \%$, the dose-response assays were performed both with and without Triton X-100. A decrease in $\mathrm{EC}_{50}$ due to Triton $\mathrm{X}-100$ is indicative of false positives due to compounds forming aggregates and sequestering proteins [15]. Two of the top hits, Disulfiram and SCH-202676, exhibited $>2$-fold reduction in levels of inhibition upon addition of $0.01 \%$ Triton X-100 - an observation consistent 
with these being aggregation-based false positives [15]. The remaining 20 compounds each gave $\mathrm{EC}_{50}$ values within the range $27 \mathrm{nM}$ to $990 \mathrm{nM}$ (Figure 5; Supplementary Table S1).

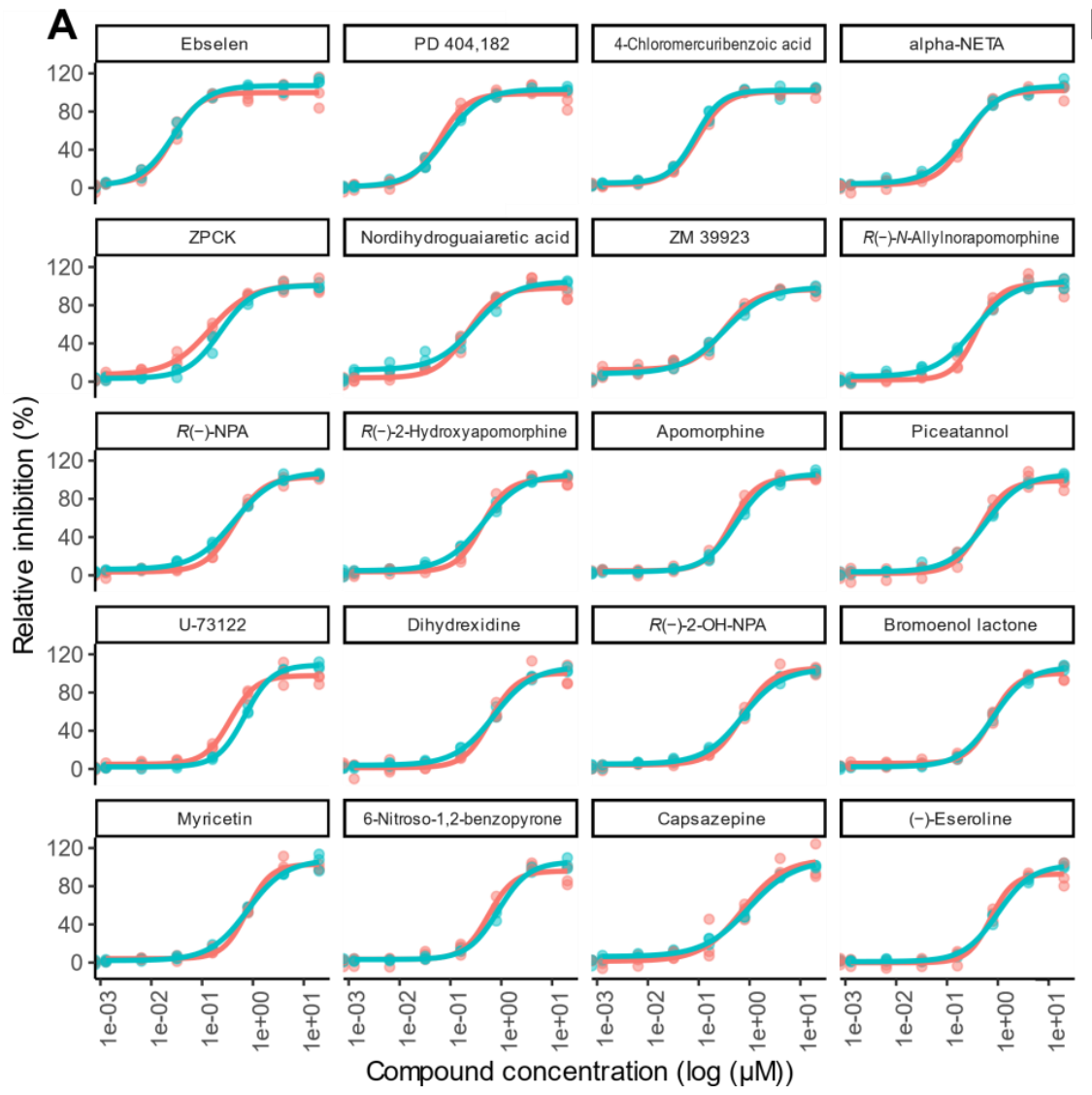

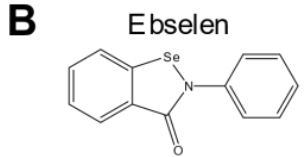

PD 404,182<smiles>N=C1Sc2ccccc2C2=NCCCN12</smiles><smiles>O=C(N[C@@H](Cc1ccccc1)C(=O)Cl)OCc1ccccc1</smiles>

Apomorphine<smiles>CN1CCc2cccc3c2[C@H]1Cc1ccc(O)c(O)c1-3</smiles>

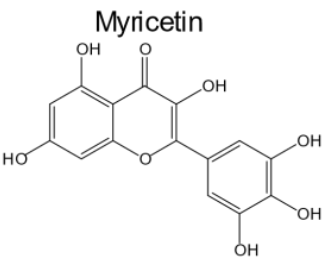

Figure 5. Dose-response curves for the top 20 inhibitors identified in this study and selected structures for compounds discussed in the text. (A) Dose-response assays were performed both with (blue) and without (red) $0.01 \%$ Triton X-100. Each compound was assessed across a 5-fold serial dilution series starting at $20 \mu \mathrm{M}$. Relative inhibition was calculated using the average $\mathrm{R}_{528 / 477}$ for the protease treated controls as $0 \%$ inhibition and the average $R_{528 / 477}$ for the no protease controls as $100 \%$ inhibition. Experiments were performed in triplicate using a different preparation of purified eCFP-Venus biosensor for each repeat. $\mathrm{EC}_{50}$ values were calculated using the R package 'dra' [16], and all data points are shown. (B) Structures of five compounds that are discussed further in the text. Compounds tested in this Panel A are: Ebselen; PD 404,182; 4-Chloromercuribenzoic acid; 2-(alpha-Naphthoyl)ethyltrimethylammonium (alpha-NETA); Z-L-Phe chloromethyl ketone (ZPCK); Nordihydroguaiaretic acid; ZM 39923; $R(-)$-N-Allylnorapomorphine; $R(-)$-Propylnorapomorphine

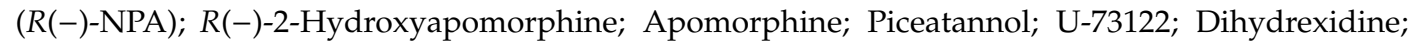
$R(-)-2,10,11-T r i h y d r o x y-N$-propylnoraporphine ( $R(-)-2-\mathrm{OH}-\mathrm{NPA}) ;$ Bromoenol lactone; Myricetin; 6-Nitroso-1,2-benzopyrone; Capsazepine; (-)-Eseroline.

\section{Discussion}

\section{Evaluation of Inhibitors Detected in This Screen}

The top hit in our screen with an $\mathrm{EC}_{50}$ of $27 \mathrm{nM}$ was ebselen, which has previously been identified as an effective inhibitor of SARS-CoV-2 3CL pro [8]. Ebselen reacts with thiol residues to form a selenosulfide bond and a general reactivity with thiols could explain its inhibitory activity against the $3 \mathrm{CL}^{\text {pro }}$ cysteine protease [17]. Supporting this presumption, another generically thiol reactive compound (4-chloromercuribenzoic acid) was the third most effective inhibitor in our assays, with an $\mathrm{EC}_{50}$ of $85 \mathrm{nM}$. Although ebselen was able to prevent viral replication in vitro [8], it has been suggested 
that its general thiol reactivity might preclude inhibition of $3 C L^{\text {pro }}$ in vivo, as the selenosulfide linkage might be reduced by cellular reactants [17]. However, ebselen appears to only covalently modify a portion of $3 \mathrm{CL}^{\text {pro }}$ [8], and molecular simulations have identified a second putative binding pocket in a region essential for dimerisation of $3 \mathrm{CL}^{\text {pro }}$. Thus, its inhibitory mechanism may not be reliant on selenosulfide bond formation [18]. While further work remains to confirm whether ebselen is a plausible therapeutic treatment, our identification of ebselen as the top hit in this study both validates our screen and supports that it is a potent inhibitor of $3 \mathrm{CL}^{\text {pro }}$.

The second strongest inhibitor identified in our screening, with an $\mathrm{EC}_{50}$ of $81 \mathrm{nM}$, was PD 404,182 . This is an antibiotic against Gram-negative bacteria that is known to inhibit 3-deoxy- $d$ manno-octulosonic acid 8-phosphate synthase [19]. PD 404,182 has also previously been shown to be a highly potent inhibitor of HCV and HIV by causing physical disruption of the virion [20,21], and its ease of synthesis suggests it is tractable for use as an antiviral [22]. It has additionally been identified as an irreversible inhibitor of dimethylarginine dimethylaminohydrolase 1 and histone deacetylase 8 via irreversible binding to cysteine residues $[23,24]$. This may suggest promiscuous binding to the cysteine residue of $3 C L^{\text {pro }}$ as a mechanism of inhibition. Nevertheless, the nanomolar activity towards $3 C^{2}$ pro is particularly potent. As PD 404,182 also has potential to disrupt virus particles, this compound might target multiple stages of the SARS-CoV-2 life cycle.

Another potentially explicable hit identified in our screen was Z-L-Phe chloromethyl ketone (ZPCK). Peptidyl chloromethyl ketones are known potent inhibitors of cysteine proteases via formation of an irreversible thioether adduct, with target specificity conferred by the peptide sequence [25]. Although mainly used for biochemical investigations, a similar strategy of linking the core structure of a peptide or peptidomimetic to a reactive warhead has successfully been used for treating hepatitis C [26] and multiple myeloma [27]. Here, ZPCK exhibited an $\mathrm{EC}_{50}$ of $230 \mathrm{nM}$, substantially lower than the analogues Tosyl-L-Phe chloromethyl ketone (TPCK; EC 50 of $1.74 \mu \mathrm{M}$ ) and Tosyl-L-Lys chloromethyl ketone (TLCK; $\mathrm{EC}_{50}$ of $10.63 \mu \mathrm{M}$ ) (Supplementary Table S1). These compounds differ from ZPCK in substituting the carboxybenzyl to a tosyl group, with an additional substitution of Phe to Lys for TLCK, both substitutions appearing to be detrimental to $3 \mathrm{CL}^{\text {pro }}$ inhibition. The ability to modulate the level of $3 \mathrm{CL}^{\text {pro }}$ inhibition by varying the peptide sequence suggests that further structure-activity-relationship studies could improve selectivity of these, and other, peptide-warhead compounds.

Five of the top twenty hits identified in our screening were apomorphine or analogues thereof (N-allylnorapomorphine, $R(-)$-propylnorapomorphine, $R(-)$-2-hydroxyapomorphine, and $R(-)-2$, 10,11-trihydroxy- $N$-propylnoraporphine). Apomorphine is an aporphine alkaloid used as a treatment for Parkinson's disease that is being explored for various additional indications including other neurological disorders, erectile dysfunction and cancer [28]. The compounds may be particularly relevant for lung infections as apomorphine can be safely administered via inhalation [29]. The five analogues identified here differ in $N$-alkylation as well as hydroxylation at the 2-position. The compounds were tolerant to both these modifications with $\mathrm{EC}_{50}$ differing at most by two-fold (346 nM to $746 \mathrm{nM}$ ). In contrast, the 10-hydroxyl group appears to be essential for activity. $R(-)$-apocodeine, which only differs from apomorphine by methylation at this position, exhibited only negligible $(6 \%)$ inhibition of $3 C L^{\text {pro }}$ in the $5 \mu \mathrm{M}$ primary screen, failing to reach the threshold for testing in dose-response assays.

Finally, whereas the plant flavonoid myricetin was found to have an $\mathrm{EC}_{50}$ of $820 \mathrm{nM}$, the related compounds quercetin, dihydroquercetin and luteolin caused only $1.9 \%, 7.5 \%$ and $1.3 \%$ inhibition, respectively, in the initial screen. Each of these flavonoids comprise a bicyclic chromone linked to a phenyl ring (the 'B-ring'), which contains three hydroxyl groups in the case of myricetin and two for quercetin, dihydroquercetin and luteolin. The additional hydroxyl is clearly important, as it is the only difference between quercetin and myricetin. Although not detected as an inhibitor at the concentrations we tested, both quercetin and its $O$-glycoside quercetin-3- $\beta$-galactoside have previously been identified as weak inhibitors of SARS-CoV 3CL ${ }^{\text {pro }}$, with $\mathrm{IC}_{50}$ values of $23.8 \mu \mathrm{M}$ and $42.8 \mu \mathrm{M}$, respectively $[30,31]$. In silico docking found the $3^{\prime}$ hydroxyl group of quercetin-3- $\beta$-galactoside forms a 
hydrogen bond with Q189 of SARS-CoV 3CL pro [31], and removal of this hydroxyl group substantially reduces inhibitory activity of both quercetin and quercetin-3- $\beta$-galactoside $[30,31]$. Being connected by a rotatable bond, the symmetry of the $B$ ring means either the $3^{\prime}$ or $5^{\prime}$ hydroxyl groups of myricetin has the potential to interact with Q189. This flexibility may explain the increased potency over quercetin, with the additional hydroxyl group increasing the likelihood of myricetin being in a conformation to form a hydrogen bond with Q189.

\section{Materials and Methods}

\subsection{Protein Expression}

Genes encoding the eCFP-Venus biosensor (Supplementary Sequence S1) and SARS-CoV-2 3CL pro (Supplementary Sequence S2) were codon-optimized and cloned into the NcoI and XhoI sites of plasmid pET28a(+) by TWIST Bioscience (Illumina; San Diego, CA, USA). The N-terminus of 3CL ${ }^{\text {pro }}$ contained the cleavage site natively found between non-structural protein 4 and $3 \mathrm{CL}^{\text {pro }}$ (TSAVLQ $\downarrow$ SG) to allow a natural terminus to form by autocleavage, and the C-terminus contained two added residues (GP) and $\mathrm{a} \mathrm{His}_{6}$-tag. The plasmids were used to transform E. coli BL21(DE3) and subsequently purified by miniprep and sequence verified by Macrogen, Inc (Seoul, South Korea).

To purify proteins, a $400 \mathrm{~mL}$ culture of $E$. coli was grown in lysogeny broth at $37^{\circ} \mathrm{C}, 200 \mathrm{rpm}$ until an $\mathrm{OD}_{600}$ of 1.6 was reached. The culture was then incubated on ice for $20 \mathrm{~min}$. Isopropyl-D-thiogalactoside (IPTG) was added at a concentration of $1 \mathrm{mM}$. The cultures were then incubated at $18^{\circ} \mathrm{C}, 200 \mathrm{rpm}$ for $18 \mathrm{~h}$ for the eCFP-Venus biosensor and $37^{\circ} \mathrm{C}, 200 \mathrm{rpm}$ for $5 \mathrm{~h}$ for $3 \mathrm{CL}^{\text {pro }}$. Cells were harvested by centrifugation and the pellet frozen. The pellet was subsequently thawed and resuspended in $30 \mathrm{~mL}$ of buffer A (20 mM Tris, $500 \mathrm{mM} \mathrm{NaCl}, 5 \mathrm{mM}$ imidazole, $\mathrm{pH}$ 8.0). For SARS-CoV-2 3CL pro the cells were lysed by sonication on ice, and for the eCFP-Venus biosensor the cells were passed twice through a French press cell disruptor (Thermo Electron; Franklin, MA, USA) at 1000 psi. The insoluble material was removed by centrifugation at $18,000 \times \mathrm{g}$ for $30 \mathrm{~min}$. The supernatant was loaded onto a column containing $2 \mathrm{~mL}$ of Ni-NTA His-Bind Resin (Novagen; Darmstadt, Germany). The column was washed with $10 \mathrm{~mL}$ of buffer A, followed by $40 \mathrm{~mL}$ of buffer A for the eCFP-Venus biosensor, or $20 \mathrm{~mL}$ of buffer B (20 mM Tris, $500 \mathrm{mM} \mathrm{NaCl}, 60 \mathrm{mM}$ imidazole, $\mathrm{pH}$ 8.0) for SARS-CoV-2 3CL ${ }^{\text {pro }}$. Bound protein was eluted using $8 \mathrm{~mL}$ of buffer $\mathrm{C}(20 \mathrm{mM}$ Tris, $500 \mathrm{mM} \mathrm{NaCl}, 1 \mathrm{M}$ imidazole, $\mathrm{pH}$ 8.0). The flow through was subjected to buffer exchange using buffer $\mathrm{D}$ ( $50 \mathrm{mM}$ Tris, $1 \mathrm{mM}$ EDTA, pH 7.5) using an Amicon Ultra 15 centrifugal filters (30 kDa cutoff, Merck Millipore) and glycerol added to a final concentration of $40 \%(v / v)$. Absorbance was measured at $280 \mathrm{~nm}$ using a NanoPhotometer ${ }^{\circledR}$ NP80 (Inplen; Munich, Germany) and used to determine the concentration based on an extinction coefficient of 49,530 M-1 $\mathrm{cm}^{-1}$ and 33,640 $\mathrm{M}^{-1} \mathrm{~cm}^{-1}$ calculated using the ExPASy ProtParam tool [32] for the eCFP-Venus biosensor and SARS-CoV-2 3CL pro, respectively.

\subsection{Enzymatic Activity and Inhibition Assays}

All assays of protease activity were performed in 96-well plate format with a total volume of $200 \mu \mathrm{L}$ of $50 \mathrm{mM}$ Tris, $1 \mathrm{mM}$ EDTA, pH 7.5. Each well contained $25 \mathrm{nM}$ of SARS-CoV-2 3CL pro and $500 \mathrm{nM}$ eCFP-Venus biosensor. Assays were set up by first adding $3 \mathrm{CL}^{\text {pro }}$ plus compound in a $100 \mu \mathrm{L}$ volume of buffer to each well, and then initiated by the addition of the eCFP-Venus biosensor in a $100 \mu \mathrm{L}$ volume. The plates were incubated at $30^{\circ} \mathrm{C}$ for $4 \mathrm{~h}$. Fluorescence was measured using an excitation wavelength of $434 \mathrm{~nm}$ and emission wavelengths of $477 \mathrm{~nm}$ and $528 \mathrm{~nm}$. High-throughput screening was performed in duplicate using $5 \mu \mathrm{M}$ of each compound. $\mathrm{Z}^{\prime}$ score was calculated using the sample means and standard deviations for the positive and negative controls. The dose-response for selected hits was performed using a concentration ranging from $0.001 \mu \mathrm{M}$ to $20 \mu \mathrm{M}$, and included a sample with no added compound. All data processing was performed in R, version 4.0.2 and scripts are available at: https://github.com/MarkCalcott/Protease_screen 


\subsection{Chemicals}

The LOPAC ${ }^{\circledR} 1280$ (International Edition) was used for screening and was purchased from Sigma-Aldrich. All compounds were supplied as stock solutions at $10 \mathrm{mM}$ and diluted in dimethyl sulfoxide to $500 \mu \mathrm{M}$ for primary screening or $1 \mathrm{mM}$ for dose-response assays.

\section{Conclusions}

This work has validated our eCFP-Venus biosensor as being robust and amenable to high-throughput screening and identified several new inhibitors of SARS-CoV-2 3CL ${ }^{\text {pro }}$. Expression levels of the biosensor were high, and each purification gave sufficient yield to screen thousands of compounds. The assay met the benchmarks for a reliable high-throughput screen, with a high $Z^{\prime}$ score, low levels of variation between replicates, and relatively few compounds interfering with the FRET signal. That the top inhibitor in this study was ebselen demonstrates the consistency of our assay with previous screening using chemically synthesised probes, as well as validating ebselen as a particularly potent inhibitor. Our screening has also identified 19 other compounds that have $\mathrm{EC}_{50}$ values below $1 \mu \mathrm{M}$, five of which were aporphine alkaloids not previously identified as $3 \mathrm{CL}^{\text {pro }}$ inhibitors, suggesting a promising starting point for structure-activity-relationship studies to develop new antiviral compounds. A key advantage of this screen over chemically synthesised probes is that it uses the same equipment that would be required for any laboratory wanting to purify and test $3 \mathrm{CL}^{\text {pro }}$, making high-throughput screening easily accessible for other researchers.

Supplementary Materials: The following are available online, Figure S1: Codon-optimized eCFP-Venus biosensor expressed from pET28a. Figure S2: Plate map showing controls for high-throughput screening. Figure S3: Dose-response curves used to calculate the $\mathrm{EC}_{50}$ values reported in Table S1. Table S1: $\mathrm{EC}_{50}$ values of the top 65 compounds identified during screening. Sequence S1: Codon-optimized eCFP-Venus biosensor with His6-tag. Sequence S2: Codon-optimised SARS-CoV-2 3CL pro. Data S1: Percentage inhibition when screening all compounds at $5 \mu \mathrm{M}$.

Author Contributions: All authors contributed to conceiving the work and writing the manuscript. Experimental work and data analysis were performed by A.S.B. and M.J.C. All authors have read and agreed to the published version of the manuscript.

Funding: This work was supported by the Royal Society of New Zealand Marsden Fund (grant 18-VUW-082 to M.J.C.) and Health Research Council of New Zealand (grant 18-532 to D.F.A.).

Conflicts of Interest: The authors declare no conflict of interest. The funders had no role in the design of the study; in the collection, analyses, or interpretation of data; in the writing of the manuscript, or in the decision to publish the results.

\section{References}

1. Menni, C.; Valdes, A.M.; Freidin, M.B.; Sudre, C.H.; Nguyen, L.H.; Drew, D.A.; Ganesh, S.; Varsavsky, T.; Cardoso, M.J.; Visconti, A.; et al. Real-time tracking of self-reported symptoms to predict potential COVID-19. Nat. Med. 2020, 26, 1037-1040. [CrossRef]

2. WHO. Coronavirus Disease (COVID-19) Dashboard. 2020. Available online: https://covid19.who.int/ (accessed on 23 September 2020).

3. Le, T.T.; Andreadakis, Z.; Kumar, A.; Roman, R.G.; Tollefsen, S.; Saville, M.; Mayhew, S. The COVID-19 vaccine development landscape. Nat. Rev. Drug Discov. 2020, 19, 305-306. [CrossRef]

4. Zhang, L.; Lin, D.; Sun, X.; Curth, U.; Drosten, C.; Sauerhering, L.; Becker, S.; Rox, K.; Hilgenfeld, R. Crystal structure of SARS-CoV-2 main protease provides a basis for design of improved alpha-ketoamide inhibitors. Science 2020, 368, 409-412.

5. Wu, F.; Zhao, S.; Yu, B.; Chen, Y.M.; Wang, W.; Song, Z.G.; Hu, Y.; Tao, Z.W.; Tian, J.H.; Pei, Y.Y.; et al. A new coronavirus associated with human respiratory disease in China. Nature 2020, 579, 265-269. [CrossRef]

6. Kelly, J.A.; Olson, A.N.; Neupane, K.; Munshi, S.; San Emeterio, J.; Pollack, L.; Woodside, M.T.; Dinman, J.D. Structural and functional conservation of the programmed-1 ribosomal frameshift signal of SARS coronavirus 2 (SARS-CoV-2). J. Biol. Chem. 2020, 295, 10741-10748. [CrossRef] 
7. Naqvi, A.A.; Fatima, K.; Mohammad, T.; Fatima, U.; Singh, I.K.; Singh, A.; Atif, S.M.; Hariprasad, G.; Hasan, G.M.; Hassan, M.I. Insights into SARS-CoV-2 genome, structure, evolution, pathogenesis and therapies: Structural genomics approach. Biochim. Biophys. Acta-Mol. Basis Dis. 2020, 1866, 165878. [CrossRef]

8. Jin, Z.; Du, X.; Xu, Y.; Deng, Y.; Liu, M.; Zhao, Y.; Zhang, B.; Li, X.; Zhang, L.; Peng, C.; et al. Structure of M-pro from SARS-CoV-2 and discovery of its inhibitors. Nature 2020, 582, 289-293. [CrossRef] [PubMed]

9. Hilgenfeld, R. From SARS to MERS: Crystallographic studies on coronaviral proteases enable antiviral drug design. FEBS J. 2014, 281, 4085-4096. [CrossRef]

10. Wu, A.; Wang, Y.I.; Zeng, C.; Huang, X.; Xu, S.; Su, C.; Wang, M.; Chen, Y.; Guo, D. Prediction and biochemical analysis of putative cleavage sites of the 3C-like protease of Middle East respiratory syndrome coronavirus. Virus Res. 2015, 208, 56-65. [CrossRef]

11. Chuck, C.P.; Chong, L.T.; Chen, C.; Chow, H.F.; Wan, D.C.; Wong, K.B. Profiling of Substrate Specificity of SARS-CoV 3CL(pro). PLoS ONE 2010, 5, e13197. [CrossRef]

12. Kilianski, A.; Mielech, A.M.; Deng, X.; Baker, S.C. Assessing Activity and Inhibition of Middle East Respiratory Syndrome Coronavirus Papain-Like and 3C-Like Proteases Using Luciferase-Based Biosensors. J. Virol. 2013, 87, 11955-11962. [CrossRef] [PubMed]

13. Nagai, T.; Miyawaki, A. A high-throughput method for development of FRET-based indicators for proteolysis. Biochem. Biophys. Res. Commun. 2004, 319, 72-77. [CrossRef] [PubMed]

14. Zhang, J.H.; Chung, T.D.; Oldenburg, K.R. A simple statistical parameter for use in evaluation and validation of high throughput screening assays. J. Biomol. Screen. 1999, 4, 67-73. [CrossRef]

15. Feng, B.Y.; Shoichet, B.K. A detergent-based assay for the detection of promiscuous inhibitors. Nat. Protoc. 2006, 1, 550-553. [CrossRef]

16. Ritz, C.; Baty, F.; Streibig, J.C.; Gerhard, D. Dose-Response Analysis Using R. PLoS ONE 2015, 10 , e0146021. [CrossRef]

17. Sies, H.; Parnham, M.J. Potential therapeutic use of ebselen for COVID-19 and other respiratory viral infections. Free Radic. Biol. Med. 2020, 156, 107-112. [CrossRef]

18. Menendez, C.A.; Bylehn, F.; Perez-Lemus, G.R.; Alvarado, W.; de Pablo, J.J. Molecular characterization of Ebselen binding activity to SARS-CoV-2 main protease. arXiv 2020, arXiv:2005.09805. [CrossRef]

19. Birck, M.R.; Holler, T.P.; Woodard, R.W. Identification of a slow tight-binding inhibitor of 3-deoxy-D-mannooctulosonic acid 8-phosphate synthase. J. Am. Chem. Soc. 2000, 122, 9334-9335. [CrossRef]

20. Chamoun, A.M.; Chockalingam, K.; Bobardt, M.; Simeon, R.; Chang, J.; Gallay, P.; Chen, Z. PD 404,182 Is a Virocidal Small Molecule That Disrupts Hepatitis C Virus and Human Immunodeficiency Virus. Antimicrob. Agents Chemother. 2012, 56, 672-681. [CrossRef]

21. Chockalingam, K.; Simeon, R.L.; Rice, C.M.; Chen, Z. A Cell Protection Screen Reveals Potent Inhibitors of Multiple Stages of the Hepatitis C Virus Life Cycle. Antivir. Res. 2010, 86, A32. [CrossRef]

22. Chamoun-Emanuelli, A.M.; Bobardt, M.; Moncla, B.; Mankowski, M.K.; Ptak, R.G.; Gallay, P.; Chen, Z. Evaluation of PD 404,182 as an Anti-HIV and Anti-Herpes Simplex Virus Microbicide. Antimicrob. Agents Chemother. 2014, 58, 687-697. [CrossRef] [PubMed]

23. Ghebremariam, Y.T.; Erlanson, D.A.; Cooke, J.P. A Novel and Potent Inhibitor of Dimethylarginine Dimethylaminohydrolase: A Modulator of Cardiovascular Nitric Oxide. J. Pharmacol. Exp. Ther. 2014, 348, 69-76. [CrossRef] [PubMed]

24. Muth, M.; Jänsch, N.; Kopranovic, A.; Krämer, A.; Wössner, N.; Jung, M.; Kirschhöfer, F.; Brenner-Weiß, G.; Meyer-Almes, F.J. Covalent inhibition of histone deacetylase 8 by 3,4-dihydro-2Hpyrimido[1,2-c][1,3]benzothiazin-6-imine. Biochim. Biophys. Acta-Gen. Subj. 2019, 1863, 577-585. [CrossRef] [PubMed]

25. Powers, J.C.; Asgian, J.L.; Ekici, Ö.D.; James, K.E. Irreversible inhibitors of serine, cysteine, and threonine proteases. Chem. Rev. 2002, 102, 4639-4750. [CrossRef]

26. Pawlotsky, J.M. Treatment of Chronic Hepatitis C: Current and Future. Hepatitis C Virus: From Mol. Virol. Antivir. Ther. 2013, 369, 321-342.

27. Groen, K.; Van De Donk, N.W.; Stege, C.A.; Zweegman, S.; Nijhof, I.S. Carfilzomib for relapsed and refractory multiple myeloma. Cancer Manag. Res. 2019, 11, 2663-2675. [CrossRef]

28. Auffret, M.; Drapier, S.; Verin, M. New tricks for an old dog: A repurposing approach of apomorphine. Eur. J. Pharmacol. 2019, 843, 66-79. [CrossRef] 
29. Grosset, K.A.; Malek, N.; Morgan, F.; Grosset, D.G. Phase IIa randomized double-blind, placebo-controlled study of inhaled apomorphine as acute challenge for rescuing 'off' periods in patients with established Parkinson's disease. Eur. J. Neurol. 2013, 20, 1445-1450. [CrossRef]

30. Ryu, Y.B.; Jeong, H.J.; Kim, J.H.; Kim, Y.M.; Park, J.Y.; Kim, D.; Naguyen, T.T.; Park, S.J.; Chang, J.S.; Park, K.H.; et al. Biflavonoids from Torreya nucifera displaying SARS-CoV 3CL(pro) inhibition. Bioorg. Med. Chem. 2010, 18, 7940-7947. [CrossRef]

31. Chen, L.; Li, J.; Luo, C.; Liu, H.; Xu, W.; Chen, G.; Liew, O.W.; Zhu, W.; Puah, C.M.; Shen, X.; et al. Binding interaction of quercetin-3-beta-galactoside and its synthetic derivatives with SARS-CoV 3CL(pro): Structure-activity relationship studies reveal salient pharmacophore features. Bioorg. Med. Chem. 2006, 14, 8295-8306. [CrossRef]

32. Gasteiger, E.; Hoogland, C.; Gattiker, A.; Wilkins, M.R.; Appel, R.D.; Bairoch, A. Protein identification and analysis tools in the ExPASy server. Methods Mol. Biol. 1999, 112, 531-552.

Sample Availability: Samples of the compounds and plasmids used in this study are available from the authors. 